\title{
Preliminary characterisation and extraction of anterior adhesive secretion in monogenean (platyhelminth) parasites
}

\author{
Tamarind E. Hamwood ${ }^{1,2}$, Bronwen W. Cribb ${ }^{3,4}$, Judy A. Halliday ${ }^{2}$, Graham C. Kearn ${ }^{5}$ and \\ Ian D. Whittington ${ }^{1}$
}

\author{
${ }^{1}$ Department of Microbiology and Parasitology, The University of Queensland, Brisbane, 4072, Australia; \\ ${ }^{2}$ Centre for Drug Design and Development, Institute for Molecular Bioscience, The University of Queensland, Brisbane, 4072 , \\ Australia; \\ ${ }^{3}$ Centre for Microscopy and Microanalysis, The University of Queensland, Brisbane, 4072, Australia; \\ ${ }^{4}$ Department of Zoology and Entomology, The University of Queensland, Brisbane, 4072, Australia; \\ ${ }^{5}$ School of Biological Sciences, University of East Anglia, Norwich, NR4 7TJ, UK
}

Key words: Platyhelminthes, Monogenea, temporary adhesion, tissue adhesion, anterior adhesive, fish ectoparasite

\begin{abstract}
Secreted anterior adhesives, used for temporary attachment to epithelial surfaces of fishes (skin and gills) by some monogenean (platyhelminth) parasites have been partially characterised. Adhesive is composed of protein. Amino acid composition has been determined for seven monopisthocotylean monogeneans. Six of these belong to the Monocotylidae and one species, Entobdella soleae (van Beneden et Hesse, 1864) Johnston, 1929, is a member of the Capsalidae. Histochemistry shows that the adhesive does not contain polysaccharides, including acid mucins, or lipids. The adhesive before secretion and in its secreted form contains no dihydroxyphenylalanine (dopa). Secreted adhesive is highly insoluble, but has a soft consistency and is mechanically removable from glass surfaces. Generally there are high levels of glycine and alanine, low levels of tyrosine and methionine, and histidine is often absent. However, amino acid content varies between species, the biggest differences evident when the monocotylid monogeneans were compared with E. soleae. Monogenean adhesive shows similarity in amino acid profile with adhesives from starfish, limpets and barnacles. However, there are some differences in individual amino acids in the temporary adhesive secretions of, on the one hand, the monogeneans and, on the other hand, the starfish and limpets. These differences may reflect the fact that monogeneans, unlike starfish and barnacles, attach to living tissue (tissue adhesion). A method of extracting unsecreted adhesive was investigated for use in further characterisation studies on monogenean glues.
\end{abstract}

Flatworm ectoparasites from the group known as monopisthocotylean monogeneans attach to their fish hosts by two mechanisms. Semi permanent attachment is mediated by a posterior haptor comprising hooks, suckers, clamps and/or glue(s) (Kearn 1994, Whittington and Combes 1994). Temporary attachment is controlled by secretion of an adhesive from the anterior end of the worm and is used when the parasites move around on host epidermis (most commonly skin and gills) (Cribb et al. 1997). Monopisthocotylean monogeneans loop over the host surface like a leech, extending and attaching the anterior end using temporary adhesive, then move the haptor forward and grip firmly with this organ, release the head end and stretch forward to attach again (Kearn 1988). The secretions involved in this temporary anterior adhesion are strong enough to be the only form of attachment on host surfaces subject to high shear forces from water currents and the adhesives act instantly and reversibly (Whittington and Cribb 2001).

In multicellular animals four types of adhesion have been defined: (a) permanent adhesion usually to abiotic substrates, as in barnacles and mussels; (b) transitory adhesion during gliding locomotion across an abiotic substrate, as in limpets; (c) temporary adhesion, as used by starfish to "walk" across an abiotic substrate; (d) temporary adhesion to living tissue (tissue adhesion), as used by monogenean parasites to attach themselves to epithelia of their fish hosts (Walker 1987, Tyler 1988, Flammang 1996, Whittington et al. 2000, Whittington and Cribb 2001). Use of tissue adhesion is unusual in the bioadhesive systems of macroorganisms and forms a unique class which is poorly studied (Whittington and Cribb 2001). In this type of adhesion the substrate may be constantly renewed, secrete mucus and generate an immune response (see Kearn 1999, Whittington et al. 2000).

Based on work on free-living "turbellarian" flatworms, Tyler (1988) has proposed a so-called "duogland" system for achieving temporary attachment and detachment. Gland ducts of two types intermingle, and Tyler hypothesised that one secretion provides the adhesive and that the other is responsible for detachment. Adhesive systems comprising two kinds of gland also occur in starfish tube feet (Flammang et al. 1998) and in the anterior adhesive areas of monogeneans such 
as the fish skin parasite Entobdella soleae (van Beneden et Hesse, 1864) Johnston, 1929 (see Whittington and Cribb 2001) but evidence from experimental work on the latter by Kearn and Evans-Gowing (1998) does not support Tyler's hypothesis. The observations on $E$. soleae suggest that both secretions, one of which consists of rod-shaped secretory bodies and the other of granular secretory bodies, are extruded together and probably interact to form the glue. "De-adhesion" (unsticking) may be brought about by the tegument through which the gland ducts pass (Kearn and EvansGowing 1998).

Only one temporary adhesive has been chemically characterised. Flammang et al. (1998) were able to provide chemical data for starfish adhesive. Although monogenean temporary adhesives have remarkable ability to bond strongly to wet, slimy and current-swept tissue surfaces, their biochemical composition is unknown, apart from the results of a few basic histochemical tests (Whittington and Cribb 2001). This is largely the result of the minute quantities produced. Consequently, a major aim of the present study was to provide a preliminary chemical characterisation of the adhesive of those monogeneans like E. soleae with two contrasting adhesive secretions and to make a comparison with chemical data from other invertebrate glues, within the confines of the limited material available. Considerable methodology was utilised to determine which techniques would enable more comprehensive characterisation when greater amounts of material became available. Isolation of adhesive material from monogeneans was also investigated with the aim of harvesting larger quantities than those available from naturally secreted material, for use in future studies.

\section{MATERIALS AND METHODS}

\section{Collection and culture of parasites and adhesive material}

Live monogeneans of the following species were collected at Heron Island, Queensland, Australia $\left(23^{\circ} 27^{\prime} \mathrm{S}, 151^{\circ} 55^{\prime} \mathrm{E}\right)$ : Merizocotyle icopae Beverley-Burton et Williams, 1989, Neoheterocotyle rhinobatidis (Young, 1967) Chisholm, 1994 and Troglocephalus rhinobatidis Young, 1967 (Monocotylidae) from the gill cavities of the common shovelnose ray, Rhinobatus typus Bennett; Entobdella sp. (Capsalidae) from the skin, Monocotyle helicophallus Measures, BeverleyBurton et Williams, 1990 and M. spiremae Measures, Beverley-Burton et Williams, 1990 from the gills and Merizocotyle australensis (Beverley-Burton et Williams, 1989) Chisholm, Wheeler et Beverley-Burton, 1995 (Monocotylidae) from nasal tissue of the pink whip ray, Himantura fai Jordan et Seale; Entobdella australis Kearn, 1978 (Capsalidae) from the skin of the blue spotted fantail ray, Taeniura lymma (Forsskål). Specimens of the skin parasite Entobdella soleae were grown on the common sole, Solea solea (L.), in aquaria at the University of East Anglia, Norwich, UK. Approximately 900 whole E. soleae specimens (between 2 and $5 \mathrm{~mm}$ long) were frozen in liquid nitrogen and transported in a dry shipper to Brisbane, Australia.

Not all species were available throughout the year of this study and the batch size of worms was restricted by what could be collected or reared. Therefore all methods/ experiments were not performed on all flatworm species. Adhesive was collected in two forms: secreted adhesive material (SAM) released naturally by the worm and presecreted extruded adhesive material (EAM) which was taken from the worm tissue before it was released.

Locomotion of live worms across glass surfaces was observed using a stereo dissecting microscope. Each parasite that attached by the anterior end left a small amount of secretion (adhesive-print) applied to the glass surface that is visible after worm detachment, using incident light. The presence of such "footprint" material was first observed by Kearn and Evans-Gowing (1998) for E. soleae. This SAM from monogenean species from Heron Island was collected for analysis on clean coverslips and slides, left unfixed and stored at room temperature until used. SAM from E. soleae was either fixed in $10 \%$ buffered neutral formalin or left unfixed. Prior to collection of SAM, coverslips and slides were washed in $100 \%$ methanol then rinsed in filtered seawater (FSW; filtered using Whatman no. 1 filter paper).

\section{Histochemistry}

SAM from E. soleae, whole thawed worms (containing unreleased adhesive) and controls (human blood smears and regions of whole thawed $E$. soleae) were exposed to alcian blue as per Humason (1972), periodic acid/Schiff's solution (PAS) and Sudan black B as per Bancroft and Stevens (1996) and stained for dihydroxyphenylalanine (dopa) as per Waite and Benedict (1984). SAM from E. soleae was also exposed to Coomassie blue and bovine serum albumen used as a control.

Solubility and proteolytic cleavage of adhesive material

Specific SAM prints of E. soleae and, when sufficient material became available, the other species listed above, were circled using a diamond pen for ease of identification on pieces of glass slide under a stereo dissecting microscope. Slides were examined using an Olympus BX60 System microscope and photographed digitally using Scion Image. These images served as an untreated reference for comparison of the visual characteristics of specific SAM prints after each solvent treatment. Three different groups of solutions were tested: protein solubilisation buffers; acids and bases; organic solvents. SAM was incubated in protein buffers at $95^{\circ} \mathrm{C}$ for at least $5 \mathrm{~min}$ and stored at $25^{\circ} \mathrm{C}$ for at least $48 \mathrm{~h}$. Protein buffers used were: sodium dodecyl sulphate reducing buffer $(2 \%$ SDS), $5 \% \beta$-mercaptoethanol ( $\beta$-ME), $20 \%$ glycerol, $62.3 \mathrm{mM}$ Tris-hydrochloric acid (Tris-HCl), $\mathrm{pH} 6.8$ (see Laemmli 1970); urea buffer (8M urea, 0.5M dithiothreitol (DTT), $0.05 \mathrm{M}$ Tris- $\mathrm{HCl}, \mathrm{pH} 8.0$ ); solubilisation buffer ( $8 \mathrm{M}$ urea, $0.5 \mathrm{M}$ DTT, $0.8 \mathrm{M}$ acetic acid); protein solubilisation buffer (6M urea, 2\% SDS, 5mM tributyl phosphine (TBP), 2\% Tris$\mathrm{HCl}$, pH 8.8) (see Molloy et al. 1998); limpet glue solubilisation buffer (1\% SDS, 1\% DTT) (see Smith et al. 1999a); and starfish glue solubilisation buffer (6M guanidine hydrochloride, $0.5 \mathrm{M}$ DTT, $0.05 \mathrm{M}$ Tris-HCl, $\mathrm{pH}$ 8.0) (see Flammang et al. 1998). Acids and bases were pipetted repeatedly over SAM and incubated at $25^{\circ} \mathrm{C}$ for at least $5 \mathrm{~min}$. 
Acids and bases tested were $80 \%$ formic acid, $1 \mathrm{M}$ sodium hydroxide and 3\% acetic acid. SAM was incubated in other solvents at $25^{\circ} \mathrm{C}$ for at least $48 \mathrm{~h}$. Solvents tested by this method were: distilled water; $100 \%$ dimethyl sulphoxide; $100 \%$ dimethyl formamide; $20 \%, 60 \%$ and $100 \%$ acetonitrile; $70 \%$ and $100 \%$ methanol; $100 \%$ acetone; $100 \%$ ethyl acetate; $100 \%$ chloroform; $100 \%$ tetrahydrofuran; $100 \%$ isopropanol; $100 \%$ 1-butanol; $100 \%$ carbon tetrachloride; $100 \%$ hexane; and $96 \%$ ethanol. After removal of each test solution, pieces of glass slide were rinsed in distilled water and air dried before being viewed and photographed as described above.

Proteolytic cleavage trials were also undertaken using SAM as above. SAM from E. soleae was treated with proteolytic enzymes. Two digests were conducted, the first using proteinase $\mathrm{K}\left(1 \%\right.$ proteinase $\mathrm{K}, 1 \mathrm{mM} \mathrm{CaCl}_{2}, 0.5 \% \mathrm{SDS}$, $0.01 \mathrm{M}$ Tris, $\mathrm{pH}$ 7.8: modified from Sambrook et al. 1989) and the second using trypsin (5\% N-tosyl-phenylalanine chloromethyl ketone [TPCK] trypsin in water) by incubating glass pieces bearing SAM for $72 \mathrm{~h}$ at $37^{\circ} \mathrm{C}, 80 \%$ humidity. Disulfide bond cleavage (adapted from Prescott 1997) was attempted by boiling SAM in $8 \mathrm{M}$ urea, $150 \mathrm{mM}$ DTT, adding iodoacetamide to a final concentration of $800 \mathrm{mM}$ and incubating at $25^{\circ} \mathrm{C}$ for $30 \mathrm{~min}$.

\section{Isolation of adhesive material}

Each SAM print from a monogenean provides a minute amount of material. The head region is a source of much greater quantities of pre-secreted adhesive. The following protocol for extraction of this pre-secreted EAM was developed through experimentation. After removal from storage in liquid nitrogen, batches of 100 E. soleae specimens were dissected. Each specimen was cut into four pieces, the head region, pharynx, body and haptor. The posterior haptor was discarded. The parasite was severed both anterior and posterior to the pharynx to separate this highly glandular organ from the head and body. The head consisted of the two laterally situated adhesive pads and the region between them, which is packed with anterior adhesive gland cells (Kearn 1994, fig. 2A). The heads and the bodies (controls) were placed separately in cavity dishes containing $1.25 \%$ sodium hypochlorite (bleach). When tissue appeared mostly disintegrated, solutions were transferred separately to microfuge tubes. Residues were washed five times by vortexing, centrifugation in a microfuge at $13000 \mathrm{rev} \cdot \mathrm{min}^{-1}$, removal of supernatant and addition of deionised water. Resulting extracted adhesive material (EAM) from the heads and bleach-treated body material (controls) were either dried using a Savant speedivac concentrator SVC100H and stored at $-20^{\circ} \mathrm{C}$ until use or processed for transmission electron microscopy. On average, $0.33 \mathrm{~g}$ of EAM was obtained from each batch of $100 \mathrm{E}$. soleae heads.

\section{Scanning and transmission electron microscopy}

Specimens of Troglocephalus rhinobatidis, Entobdella sp. and Neoheterocotyle rhinobatidis were fixed in $10 \%$ phosphate buffered neutral formalin for studies of the anterior adhesive area morphology. SAM from these species was collected on glass and fixed in $10 \%$ formaldehyde for observation. All samples were washed in cacodylate buffer $(0.1 \mathrm{M}$ cacodylate, $0.15 \mathrm{M}$ sucrose, $\mathrm{pH} 7.2)$ and post-fixed at $25^{\circ} \mathrm{C}$ in $1 \%$ osmium tetroxide in cacodylate buffer. After dehydration in a graded ethanol series, specimens were infiltrated with hexamethyldisilazane and allowed to dry by evaporation (see Whittington and Cribb 1998). SAM from $E$. soleae was either fixed in $10 \%$ buffered neutral formalin or left unfixed and dried by evaporation. All samples were mounted on aluminium stubs using double-sided adhesive tape and silver conductive paint, coated with gold to a thickness of $7-10 \mathrm{~nm}$ and viewed using a JEOL $6300 \mathrm{~F}$ scanning electron microscope operating at $7-10 \mathrm{kV}$.

A pellet of EAM from E. soleae was suspended in $2 \%$ agarose and fixed in 3\% glutaraldehyde in cacodylate buffer. Adult $E$. soleae were fixed in $3 \%$ glutaraldehyde in $0.1 \mathrm{M}$ cacodylate buffer in filtered seawater (FSW). Both samples were then washed in cacodylate buffer and post-fixed in $1 \%$ osmium tetroxide in cacodylate buffer, dehydrated in a graded series of acetone, infiltrated with Spurr resin and polymerised at $60^{\circ} \mathrm{C}$ for 3 days. Sections were cut using a diamond knife, stained with uranyl acetate and lead citrate and examined using a JEOL 1210 transmission electron microscope operating at $80 \mathrm{kV}$.

\section{Analytical methods}

Batches of worm regions were analysed and compared using SDS polyacrylamide gel electrophoresis (PAGE) and iso-electric focussing (IEF). Material comprised 100 heads (containing adhesive) or 100 bodies (minus adhesive region, pharynx and haptor: control) of E. soleae. Samples for SDS PAGE were either treated with bleach (see Isolation of adhesive material above) or vortexed and sonicated in protein solubilisation buffer (buffer as per Molloy et al. 1998). Batches of $20 \mathrm{E}$. australis heads and bodies were also prepared for SDS PAGE by treatment in $0.05 \%$ Brij-35 with glass beads followed by freezing, thawing, vortexing, centrifuging and sonication. The procedure was repeated and then samples were dried in a Savant speedivac concentrator SVC100H and ground using a mortar and pestle. A BioRad protein assay sensitive to $1.2 \mu \mathrm{g}$ protein was used to quantify the amount of protein present. Samples and molecular weight standards were analysed on $16 \% \mathrm{~T}$ SDS PAGE with a $4 \%$ stacking gel, run at $150 \mathrm{~V}$ for $1 \mathrm{~h}$. For IEF, head and body regions of E. soleae were ground in liquid nitrogen using a mortar and pestle. Samples and IEF standards were run on a $6 \% \mathrm{~T}$ IEF gel $\mathrm{pH} 4-8$ at $100 \mathrm{~V}$ for $1 \mathrm{~h}, 250 \mathrm{~V}$ for $1 \mathrm{~h}$ and $500 \mathrm{~V}$ for $1 \mathrm{~h}$. Where the amount of protein was undetectable using colloidal Coomassie stain (for $30 \mathrm{~min}$, then destained overnight), gels were silver stained according to the protocol of Johansson and Skoog (1987).

SAM was insoluble in all but one buffer $(6 \mathrm{M}$ guanidine hydrochloride, $0.5 \mathrm{M}$ DTT, $0.05 \mathrm{M}$ Tris- $\mathrm{HCl}, \mathrm{pH} 8.0$ ) and this was incompatible with electrophoretic techniques. When solution was injected using high pressure liquid chromatography (HPLC), the buffer became diluted in chromatography buffers and the column became obstructed, suggesting that the protein precipitated into an insoluble form. For other HPLC, thawed, homogenised heads and median body regions, and proteinase K-digested SAM and control samples of E. soleae were used. Samples were halved and one half was suspended in Brij- 35 and run on a VYDAC C-18 $(4.6 \mathrm{~mm} \times 250 \mathrm{~mm}, 5$ $\mu \mathrm{m}, 300 \AA)$ analytical column, $0-100 \%$ solvent $(90 \%$ (v/v) acetonitrile in $0.09 \%(\mathrm{v} / \mathrm{v})$ TFA) gradient at $1 \mathrm{ml} / \mathrm{min}$ and fractions collected. The other half was suspended in $20 \%$ acetonitrile and run as above, with $20-100 \%$ solvent $(90 \%$ 
acetonitrile/0.05\%TFA) gradient. Fractions were stored at $-20^{\circ} \mathrm{C}$. Chromatography was conducted on a Hewlett Packard series 1100 HPLC pump system with a degasser, quaternary pump, autosampler, diode array detector set to $215 \mathrm{~nm}$ using Hewlett Packard Chemstation software on a Hewlett Packard Kayak XA computer.

EAM and bleach-treated bodies from $50 \mathrm{E}$. soleae specimens were dried using a speedivac, resuspended and digested in $5 \mu \mathrm{g} / \mathrm{ml}$ trypsin buffered in $1 \mathrm{M}$ ammonium bicarbonate overnight at $37^{\circ} \mathrm{C}$. A control digestion, with no sample present, was conducted for comparison of trypsin autodigestion with digests of sample. The samples were run on HPLC using a ZORBAX C-18 column $(50 \mathrm{~mm} \times 210 \mathrm{~mm}, 3.5$ $\mu \mathrm{m}, 300 \AA$ ) under the same conditions as for the undigested $E$. soleae heads and bodies above.

Head and body samples of E. soleae for liquid chromatography-mass spectroscopy (LCMS) were prepared as for HPLC and concentrated in a speedivac before injection using an Applied Biosystems 112A oven/injector onto a PE SCIEX API III LCMS with Perkin Elmer ABI 140D solvent delivery system with a Brownlee C18 $(0.5 \mathrm{~mm} \times 150 \mathrm{~mm}, 5 \mu \mathrm{m}, 300$ $\AA)$ column. The gradient was $0-60 \%$ solvent $(90 \%(\mathrm{v} / \mathrm{v})$ acetonitrile in $0.09 \%(\mathrm{v} / \mathrm{v}) \mathrm{TFA})$ at a flow rate of $1 \mathrm{ml} / \mathrm{min}$.

SAM from about 100 adhesive-prints of Monocotyle helicophallus, M. spiremae, Merizocotyle australensis, M. icopae, Neoheterocotyle rhinobatidis, Troglocephalus rhinobatidis and E. soleae and EAM from E. soleae were analysed for amino acid content. The SAM was washed in $70 \%$ ethanol before processing to remove contaminating proteins but removal of all background is not possible so a control was run with $E$. soleae to determine the level of background present. Washing was unnecessary for EAM samples. All samples were dried in vacuo and incubated in vacuo at $150^{\circ} \mathrm{C}$ for 45 min with $6 \mathrm{M}$ hydrochloric acid $(\mathrm{HCl}) . \mathrm{HCl}$ was removed by vacuum, amino acids were taken up in $20 \%$ acetonitrile and transferred to a microfuge tube. Amino acids were dried and stored at $-20^{\circ} \mathrm{C}$. The residue was resuspended in acetonitrile before being labelled fluorescently as per Cohen and Michaud (1993). Reaction components were vortexed and stored at $37^{\circ} \mathrm{C}$ for $30 \mathrm{~min}$ to remove by-products. Samples were then loaded onto a Waters AccQ-Tag column in a Waters 600 HPLC system connected to a Perkin Elmer series 200 LC pump and a 490 Waters fluorescent detector. The buffer system comprised solvent A $(0.1 \%(\mathrm{v} / \mathrm{v})$ trifluoroacetic acid (TFA)) and solvent B (90\% (v/v) acetonitrile in $0.09 \%(\mathrm{v} / \mathrm{v})$ TFA) run with a $1 \%$ gradient at $1 \mathrm{ml} / \mathrm{min}$. The concentration of individual amino acids was calculated by comparison of peak size to the peak of the internal standard $(\alpha-\mathrm{ABA})$ of known concentration.

\section{Trials to inhibit adhesion}

Live specimens of $T$. rhinobatidis and $N$. rhinobatidis were used to determine whether heparin inhibits adhesion. Living monogeneans were placed into glass cavity blocks containing FSW and 300U/ml heparin; the control contained FSW. For 6 $\mathrm{h}$, all specimens were examined every $30 \mathrm{~min}$ for anterior attachment by prodding worms with a metal needle to induce movement. Specimens were also examined after $24 \mathrm{~h}$.

\section{RESULTS}

\section{Secreted Adhesive Material (SAM) morphology}

Using SEM, SAM of the monogenean E. soleae was visible as paired structures (Fig. 1A). Fine structure of monogenean SAM was seen to vary greatly between species. The homogeneous matrix of SAM from $E$. soleae (Fig. 1B) bears little resemblance to the net-like structure of SAM from T. rhinobatidis (Fig. 1C). The SAM from E. soleae (Fig. 1B) has extraneous material on its surface that is not apparent on the adjacent glass substrate, but washes off in water. There is no greater similarity between closely related species, e.g. between the monocotylids $T$. rhinobatidis and $N$. rhinobatidis (image not shown), and between the capsalids $E$. soleae and Entobdella sp. (image not shown), than between less closely related species, e.g. E. soleae and $T$. rhinobatidis.

Using fine forceps, a line could be drawn through the SAM on a glass surface indicating it has a viscous, rather than hard or brittle composition. SAM could also be scraped off the glass substrate.

\section{Histochemistry}

SAM of E. soleae stained positively with Coomassie blue. It did not react with periodic acid/Schiff's reagent (PAS), alcian blue, or Sudan black B. SAM was also negative for dopa. The anterior adhesive pads of whole specimens of E. soleae were positive for PAS. These regions stained poorly with Sudan black B in contrast to the general body area which stained more strongly. Anterior adhesive areas were also weakly positive for alcian blue. No discrete adhesive bodies or duct contents were observed to stain more densely than the surrounding tissues. The anterior adhesive areas were negative for dopa, as was the body tissue except for the vitellarium of the reproductive system and the eggs. Positive controls indicated that methodology and reagents were sound.

\section{Solubility, proteolytic cleavage and isolation}

SAM, principally tested from $E$. soleae, was found to be insoluble in all acids and bases used, in all organic solvents used and in all but one protein solubilisation buffer. Some solubility was achieved using $6 \mathrm{M}$ guanidine hydrochloride, $0.5 \mathrm{M}$ dithiothreitol, $0.05 \mathrm{M}$ Tris- $\mathrm{HCl}, \mathrm{pH} 8.0$ (i.e. the starfish glue solubilisation buffer of Flammang et al. 1998).

SAM from E. soleae was digested completely using proteinase $\mathrm{K}$ and trypsin. When these treated adhesiveprints were viewed using SEM, all structural integrity was destroyed. Iodoacetamide-mediated disulphide cleavage (cysteine reduction and alkylation) showed inconsistent solubilisation of E. soleae SAM; some prints were removed, but others remained.

A technique to isolate putative pre-secreted adhesive material by bleach disintegration of surrounding, nonadhesive tissue was developed for E. soleae. During 


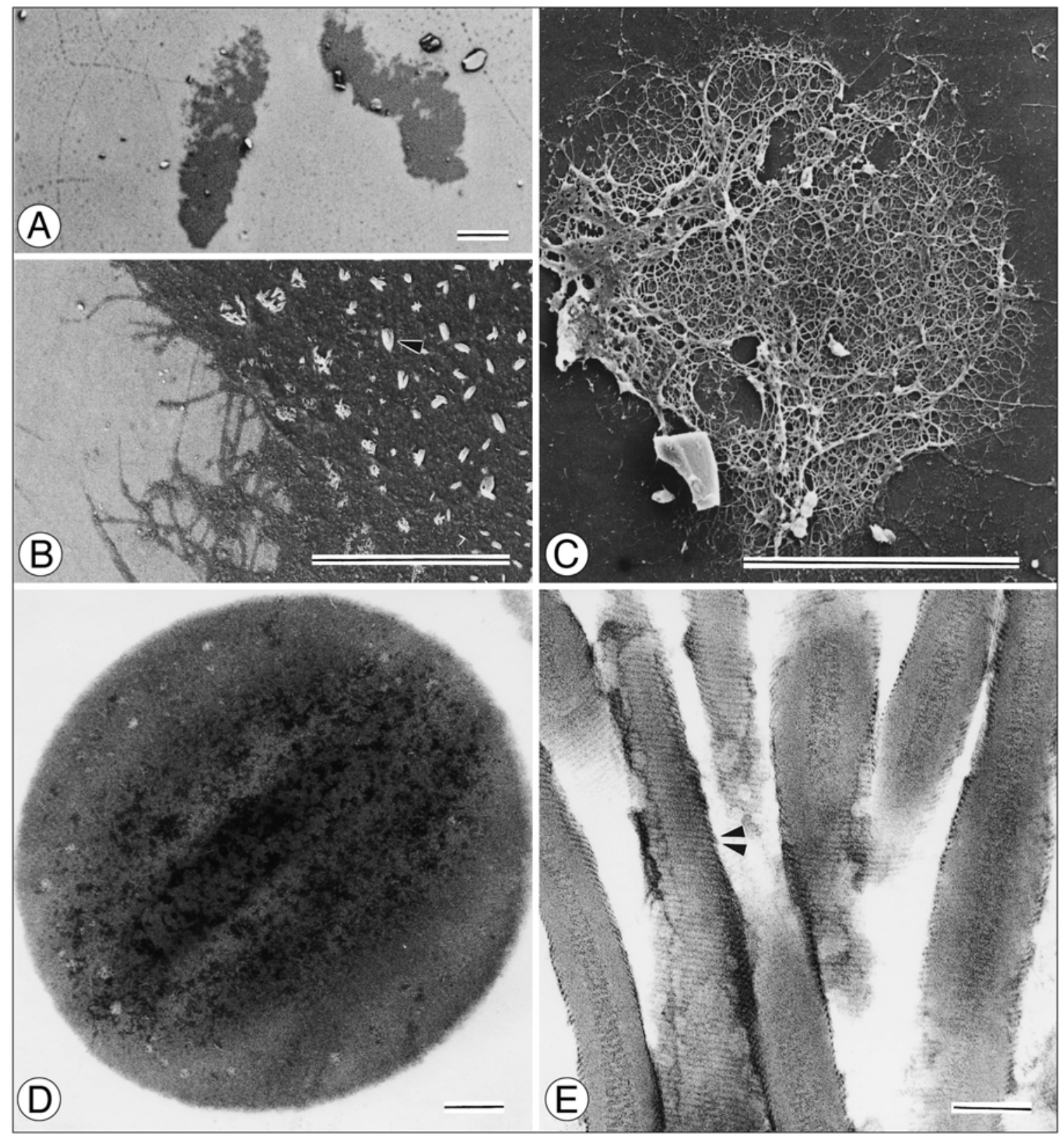

Fig. 1. Adhesive secretion from the anterior ends of some monogenean parasites. $\mathbf{A}$ - scanning electron micrograph of a pair of adhesive-prints (SAM) from a single specimen of Entobdella soleae (Capsalidae); B - detail of SAM from E. soleae showing a homogeneous matrix and extraneous material on its surface (arrowhead); $\mathbf{C}$ - scanning electron micrograph of SAM prints from Troglocephalus rhinobatidis (Monocotylidae) showing rod-like secretion forming long strands; D - transmission electron micrograph of extracted material (EAM) from E. soleae; $\mathbf{E}$ - transmission electron micrograph showing a longitudinal section of rod-like secretory bodies from $E$. soleae, with internal banding (arrowheads). Scale bars: A $=100 \mu \mathrm{m} ; \mathrm{B}, \mathrm{C}=10 \mu \mathrm{m} ; \mathrm{D}, \mathrm{E}=200$ $\mathrm{nm}$.

disintegration of worm tissue, spherical bodies with high contrast developed in the anterior adhesive areas when observed using phase contrast microscopy. The spheres appeared in the region where the rod-like secretory bodies are found and eventually floated free in the bleach solution as the surrounding tissue disintegrated. No spheres were seen in other body regions treated similarly. This bleach-isolated extracted adhesive material (EAM, Fig. 1D) appeared similar in electron density and granularity to the unsecreted rod- 
like bodies viewed in thin sections of intact worms (Fig. 1E). There were also many similarities between the amino acid content of EAM and SAM from E. soleae (see below and Table 1).

\section{SDS, IEF, HPLC and LCMS}

No differences were observed between heads and body samples of $E$. soleae using either SDS PAGE or IEF. Neither technique was successful, however, because the adhesive proteins were insoluble (see above) and therefore unable to enter the matrix of the gel and so remained in the wells. This was particularly apparent in the IEF gel where wells stained heavily for protein. Similarly, SDS PAGE was unsuccessful for $E$. australis.

Some minor differences were apparent between HPLC chromatograms of aqueous and acetonitrile extracts of heads and bodies of $E$. soleae, but no peaks were large enough for purification. With LCMS, it was not possible to differentiate peaks from background; peaks were too small for analysis for molecular weight determination. Chromatograms of EAM and bleachtreated body of $E$. soleae showed no significant differences. Also, no significant difference was found between proteinase K-digested E. soleae SAM and proteinase K. However, trypsin-digested EAM showed a peak which eluted at about $25 \%$ solvent which was absent in the autolyzed trypsin control and the trypsindigested body of $E$. soleae.

\section{Amino acid content}

Amino acid analysis of SAM from seven species of monogeneans was conducted. It might be considered that SAM scraped from glass surfaces exposed to seawater would yield unrepresentative adhesive composition due to the acquisition of a natural background of adsorbed proteins and glycoproteins onto the glass surface. This background was investigated for $E$. soleae and only contributed a mean of 5 residues/1000 (range $0-13)$ to values obtained for E. soleae SAM.

Across the seven species of monogeneans, generally there were high levels of glycine (12-19\%) and alanine (6-14\%) whereas tyrosine and methionine were found at low levels (1-3\% and $0-2 \%$ respectively), and histidine was often absent (Table 2). However, amino acid content varied across species. Some differences were apparent when species were divided along family lines. Only two of the 16 amino acid residue values (serine and arginine) for the capsalid, E. soleae, show an overlap with the range of values seen for the six monocotylid species. Also, E. soleae showed a noticeably lower amount of leucine and phenylalanine (Table 2: $6 \%$ vs. $12 \%$ and $3 \%$ vs. $11 \%$ respectively) than five of the six monocotylid species. Within the monocotylids, Merizocotyle australensis differed from
Table 1. Amino acid composition of secreted adhesive material (SAM), extracted adhesive material (EAM) and body taken from Entobdella soleae (Es). Values in residues per 1000

\begin{tabular}{|l|c|c|c|}
\hline & \multicolumn{3}{|c|}{ Entobdella soleae } \\
Amino acid & Body & EAM & SAM \\
\hline Asparagine or aspartate (Asx) & 0 & 111 & 98 \\
Serine & 43 & 53 & 75 \\
Glutamine or glutamate (Glx) & 0 & 182 & 144 \\
Glycine & 76 & 124 & 117 \\
Histidine & 0 & 1 & 16 \\
Arginine & 9 & 0 & 43 \\
Threonine & 20 & 7 & 53 \\
Alanine & 426 & 115 & 64 \\
Proline & 98 & 108 & 45 \\
Tyrosine & 104 & 6 & 27 \\
Valine & 91 & 92 & 102 \\
Methionine & 0 & 0 & 17 \\
Lysine & 15 & 36 & 73 \\
Isoleucine & 57 & 70 & 40 \\
Leucine & 47 & 79 & 61 \\
Phenylalanine & 12 & 16 & 26 \\
\hline
\end{tabular}

the other five species in having higher amounts of serine, arginine and proline, while displaying lower amounts of leucine and phenylalanine. If this species is excluded, the five remaining monocotylids show a consistent amino acid composition, except that Monocotyle helicophallus has a lower level of serine $(1 \%)$ than the other four monocotylid species.

Comparison of amino acid composition for E. soleae shows that EAM differs greatly from general body tissue, but is not identical to SAM (Table 1). Both EAM and SAM contain significant amounts of the acidic residues Asx (asparagine or aspartate: 10\%) and Glx (glutamine or glutamate: 14-18\%) whereas these are absent in body tissue. EAM and SAM also contain slightly more glycine ( $12 \%$ vs. $8 \%$ in the body). Body tissue has a much higher content of alanine (43\% vs. 6$11 \%)$ and tyrosine $(10 \%$ vs. $<3 \%)$ than either EAM or SAM. However, EAM was more similar to body composition for histidine, arginine, proline, methionine and lysine.

\section{Adhesion inhibition trials}

Trials to inhibit adhesion in two monocotylid species were conducted using heparin, a glycosaminoglycan possibly involved in de-adhesion of starfish (Thomas and Hermans 1985). Complete inhibition of adhesion to glass by Troglocephalus rhinobatidis and Neoheterocotyle rhinobatidis was not achieved. All control parasites maintained in FSW remained alive and attached, able to adhere to glass for $24 \mathrm{~h}$. Only $30 \%$ of parasites maintained in FSW containing heparin remained adhering to the glass, but all remained alive for the $24 \mathrm{~h}$ duration of the experiment. 
Table 2. Amino acid composition of secreted adhesives from different marine organisms. Organisms included are the monogenean flatworms Entobdella soleae (Es), Merizocotyle australensis (Ma), M. icopae (Mi), Monocotyle spiremae (Ms), M. helicophallus (Mh), Troglocephalus rhinobatidis $(\mathrm{Tr})$ and Neoheterocotyle rhinobatidis $(\mathrm{Nr})$, the starfish echinoderm Asterias rubens (Ar), the barnacles Balanus eburneus (Be) and Megabalanus rosa $(\mathrm{Mr})$ and the limpet Lottia limatula (L1). Values in residues per 1000 .

\begin{tabular}{|c|c|c|c|c|c|c|c|c|c|c|c|}
\hline \multirow[b]{2}{*}{ Amino acid } & \multicolumn{7}{|c|}{ Monogeneans } & Starfish & \multicolumn{2}{|c|}{ Barnacles } & Limpet \\
\hline & $E s^{a}$ & $\mathrm{Ma}^{\mathrm{a}}$ & $\mathrm{Mi}^{\mathrm{a}}$ & $\mathrm{Ms}^{\mathrm{a}}$ & $\mathrm{Mh}^{\mathrm{a}}$ & $\operatorname{Tr}^{\mathrm{a}}$ & $\mathrm{Nr}^{\mathrm{a}}$ & $\mathrm{Ar}^{\mathrm{b}}$ & $\mathrm{Be}^{\mathrm{c}}$ & $\mathrm{Mr}^{\mathrm{d}}$ & $\mathrm{Ll}^{\mathrm{e}}$ \\
\hline Asparagine or aspartate (Asx) & 98 & 70 & 53 & 31 & 55 & 84 & 54 & 118 & 57 & 91 & 127 \\
\hline Serine & 75 & 149 & 82 & 84 & 10 & 80 & 76 & 76 & 83 & 99 & 90 \\
\hline Glutamine or glutamate (Glx) & 144 & 101 & 57 & 130 & 69 & 83 & 61 & 102 & 64 & 92 & 115 \\
\hline Glycine & 117 & 193 & 189 & 187 & 161 & 152 & 181 & 97 & 66 & 79 & 80 \\
\hline Histidine & 16 & 5 & 0 & 0 & 1 & 3 & 0 & 21 & 23 & 13 & 10 \\
\hline Arginine & 43 & 61 & 39 & 39 & 43 & 37 & 41 & 41 & 75 & 56 & 33 \\
\hline Threonine & 53 & 4 & 24 & 29 & 46 & 34 & 31 & 78 & 57 & 71 & 116 \\
\hline Alanine & 64 & 103 & 139 & 104 & 80 & 97 & 117 & 62 & 49 & 75 & 52 \\
\hline Proline & 45 & 67 & 54 & 58 & 53 & 53 & 56 & 61 & 86 & 49 & 79 \\
\hline Tyrosine & 27 & 14 & 16 & 11 & 15 & 14 & 16 & 27 & 75 & 42 & 18 \\
\hline Valine & 102 & 55 & 45 & 58 & 47 & 48 & 44 & 67 & 62 & 73 & 72 \\
\hline Methionine & 17 & 0 & 8 & 11 & 2 & 11 & 9 & 17 & 11 & 16 & 11 \\
\hline Lysine & 73 & 42 & 41 & 29 & 39 & 39 & 42 & 56 & 53 & 57 & 59 \\
\hline Isoleucine & 40 & 39 & 31 & 29 & 33 & 33 & 33 & 45 & 69 & 53 & 52 \\
\hline Leucine & 61 & 74 & 123 & 112 & 125 & 121 & 126 & 61 & 110 & 83 & 51 \\
\hline Phenylalanine & 26 & 31 & 101 & 112 & 129 & 111 & 113 & 38 & 53 & 37 & 26 \\
\hline Cysteine or half-cystine & $?^{*}$ & $?^{*}$ & $?^{*}$ & $?^{*}$ & $?^{*}$ & $?^{*}$ & $?^{*}$ & 32 & 6 & 16 & 20 \\
\hline
\end{tabular}

*Acid hydrolysis destroys this amino acid; ${ }^{\text {a Present work; }}{ }^{\mathrm{b}}$ Flammang et al. (1998); ${ }^{\mathrm{c}}$ Naldrett and Kaplan (1997); ${ }^{\mathrm{d}}$ Kamino et al. (1996); ${ }^{\text {e }}$ Smith et al. (1999a).

\section{DISCUSSION}

This study has provided for the first time a preliminary characterisation for the anterior adhesive secretions of some monopisthocotylean monogenean parasites (platyhelminths). This secretion is used during locomotion for temporary adhesion to living host tissue - to the lining of the gill cavity or to external skin surfaces. To date there has been no published study on the biochemical composition of any platyhelminth temporary adhesive secretion beyond some basic histochemical tests (Whittington and Cribb 2001). Only one other study, that of Flammang et al. (1998), has characterised a temporary adhesive, specifically that of the starfish Asterias rubens used for attachment of the tube feet to a non-living surface. Monogenean glue for analysis was obtained by permitting living parasites to attach to glass and harvesting the patches of glue ("footprints") left behind at sites of temporary attachment of the head. Because of the minute amount of glue in each footprint, many footprints were needed to provide sufficient material (SAM) for analysis. This remains a limitation to any study of monogenean anterior adhesives. Sodium hypochlorite (bleach) digestion, despite its drawbacks, appears to be the only technique by which unsecreted adhesive (EAM) can be harvested from whole parasites but this approach must be viewed with caution due to the likely modification of the amino acid profile caused by application of the technique.

\section{General characteristics of monogenean temporary adhesives}

The SAM from the anterior adhesive areas of the monogenean species studied here is proteinaceous because it is digested by proteolytic enzymes (proteinase $\mathrm{K}$, trypsin) and reacts with Coomassie blue, a stain sensitive to protein. On the basis of stains, SAM of Entobdella soleae does not contain polysaccharides, including acid mucins, or lipids. It is also highly insoluble. The SAM has a soft consistency, rather than becoming hard or brittle. Some of these features, such as insolubility, softness and protein content, are similar to the temporary adhesive used by the starfish Asterias rubens, but carbohydrate and lipid are also present in the adhesive of this echinoderm (Flammang et al. 1998). Other marine glues secreted by barnacles and limpets also display insolubility (Kamino et al. 1996, Smith et al. 1999a). A morphological comparison of the SAM by SEM from the monocotylid monogenean Troglocephalus rhinobatidis with that from the starfish $A$. rubens (see Flammang et al. 1998) also shows a similarity in appearance. SAM from the monogenean adhesive is a porous material comprising a network of strands; the starfish "print" has a similar, spongy appearance but this results from raised ridges of homogeneous material. As Flammang et al. (1998) point out, this is common to some other marine invertebrates: the byssal plaques of mussels (Benedict and Waite 1986, Waite 1986) and basal cement of barnacles (Naldrett 1993) also show a spongy organisation. However, in monogenean SAM, the rod-shaped secretions retain some integrity. The 
strands apparent in the SAM of the monocotylid $T$. rhinobatidis (Fig. 1C) are of the same dimension as the rod-shaped secretions. Also, although rod-shaped secretion is not obvious in the apparently homogeneous matrix of the capsalid E. soleae (Figs. 1A, B), Kearn and Evans-Gowing (1998) found degraded rod-shaped bodies which retained outer membranes within the glue matrix when viewed using transmission electron microscopy. Such retention may be important in the adhesion process. In the case of the starfish "footprint", all secretions have spherical morphology before release, and cannot be distinguished in the secreted adhesive (Flammang et al. 1998). Both types of starfish adhesive bodies (Flammang et al. 1994) and the rod-like bodies of monogeneans display regular patterns or bands akin to that presented in Fig. 1E for E. soleae (see Cribb et al. 1997, 1998, Whittington and Cribb 1998, 1999). It is possible that such banding represents a regular packing of large protein molecules.

\section{Solubilisation of adhesive}

Some solubilisation of E. soleae SAM was achieved using a buffer which was successful in solubilising starfish temporary adhesive $(6 \mathrm{M}$ guanidine hydrochloride, $0.5 \mathrm{M}$ dithiothreitol, $0.05 \mathrm{M}$ Tris- $\mathrm{HCl}, \mathrm{pH} 8.0$ : Flammang et al. 1998). Unfortunately material dissolved in this buffer could not be analysed further using HPLC due to later precipitation of the proteins. Removal of DTT by HPLC allows the disulfides to reform. To prevent this, one can alkylate the thiols using vinylpyridine or iodoacetate. This was tried, using the small amounts of secretion available, but without success. Other techniques are available, such as column chromatography using 6M GuHCL as eluant, and could be tried when larger amounts of material become available. Many protein buffers are similar in the nature of their components, each of which disrupts a different type of bond in protein conformation. Chaotropic agents (e.g. urea and guanidine hydrochloride) disrupt hydrogen bonds in the protein's environment whereas SDS and other detergents are amphipathic molecules that interact with both hydrophilic protein and the hydrophobic environment. Intra- and intermolecular disulphide bonds can be reduced using DTT, $\beta$-ME or $\mathrm{TBP}$, and $\mathrm{pH}$ can help denature proteins by breaking ionic interactions. Combinations within the buffers that we tried point to the efficacy of using guanidine hydrochloride in combination with other agents such as DTT. SDS is not effective. Optimal solubilisation was achieved when the protein had undergone proteolysis.

\section{Biochemical analyses of adhesive}

In general, analyses of monogenean adhesive material were hindered by the insolubility of the material and the size of samples obtained. Neither SDS PAGE nor IEF were successful. It might be suggested that we should have dialysed the extract against a buffer compatible with electrophoresis that contains urea but we did not do this because extracting buffers containing urea did not achieve solubility and the material was, at best, only partially soluble in guanidine hydrochloride. Our results also indicate that EAM, like SAM, is insoluble under most conditions. However, with further study when more worms become available, it will be a promising line of enquiry to increase sample size and repeat the trypsin digestion of EAM and SAM using HPLC for analysis because this showed a peak unique to the EAM. In this preliminary characterisation of monogenean anterior adhesive, some amino acid analysis was achieved. To allow for a more complete analysis of the material such as determination of percentage protein and confirmation of the absence of lipids and carbohydrates (as indicated by our histochemical tests), it will be necessary to collect a greater quantity of SAM than was available for this study. Lectins could also be used to study the chemistry of the anterior adhesive area duct endings and associated specialised tegument (see Whittington et al. 2000) to probe for glycoproteins and glycosaminoglycans.

Amino acid analysis of SAM and EAM from the capsalid monogenean E. soleae demonstrates distinct differences from body tissue. EAM is not identical to SAM. Comparison must be viewed with caution because the methodology will oxidise amino acids without alkyl side chains and differences are apparent in histidine, arginine, methionine and lysine percentages (see Table 1), being amino acids most prone to oxidation by hypochlorite. Some other differences may be due to contaminant proteins that were not completely removed by the extraction process. Alternatively, the SAM may contain material from the tegument and/or spherical secretory bodies that perhaps may be absent from EAM. If antigenicity is retained in EAM then immunocytochemistry may resolve this. Development of an antibody to the EAM and its use to label sites in the worms as well as in SAM would test the identity of EAM as the anterior adhesive material. Labelling would also indicate whether one or both types of adhesive bodies present in the anterior adhesive area of E. soleae are present in EAM. It seems highly likely that EAM is the anterior adhesive material because rod-like secretion is proteinaceous and insoluble: in a previous study reported by Whittington and Cribb (2001), the rod-like secretion of $E$. soleae was partially digested in situ by proteinase $\mathrm{K}$. These rod-like bodies were also found to be much more resistant to solubilisation or digestion than the surrounding anterior tissue (unpublished). Homology between EAM and rods is supported by the TEM evidence that shows similar electron density and granularity for EAM and rod-like bodies (Figs. 1D, E).

With hypochlorite digestion there is always the risk of producing an extract that is significantly changed in functionality and antigenicity. At present there is no other technique available for extracting any significant amount of adhesive material from the anterior end of monogeneans so the limitations of the methodology 
must be tolerated. However our observations that the adhesive is highly insoluble in situ, possibly tightly packed and forms spherules of material as the surrounding tissue disintegrates, suggests that much of the adhesive may remain unaffected. While not optimal for characterisation, with careful timing, the material harvested from this technique may be able to be used to raise antibodies, yield some sequence data and develop specific DNA primers or molecular probes.

The adhesive in E. soleae does not contain dopa, the substrate of quinone tanning in the adhesive mechanism proposed for mussel glues (Waite 1983, 1990). Using histochemistry, dopa was absent in SAM and the in situ pre-secreted adhesive although the test was specific for $o$-diphenolic residues. This absence is not surprising because of the fast-acting nature of the adhesive in monogeneans. In contrast, the glues of mussels take time to produce thread and plaque and so 2 to $5 \mathrm{~min}$ is needed for construction, and these adhesives cure over a few hours (Rzepecki and Waite 1995).

The amino acid composition of SAM is variable amongst the seven monogenean species studied and may be unique to each species. Generally there are high levels of glycine and alanine, low levels of tyrosine and methionine, and histidine is often absent. Similarities in concentrations of specific amino acids are apparent in most of the closely related species, but Merizocotyle australensis stands apart from the other five monocotylid species investigated. There is a possible difference at family level when the capsalid, E. soleae, is compared to the monocotylids (Table 2), but the Capsalidae is represented by only one species in our analysis. The SAM of the monogeneans studied here shows an overall similarity in amino acid content with starfish temporary adhesive, barnacle cement and limpet transitory adhesive in that the ranges of residue values overlap (Table 2). Some individual amino acids vary noticeably between monogenean species and the other marine organisms: e.g., glycine is present in higher concentration and threonine is at a lower concentration in the monogeneans, especially when compared with starfish and limpets. Isoleucine is also present at a lower level in monogeneans. It is not surprising that there is a difference between these organisms because starfish and limpets adhere to abiotic surfaces whereas monogeneans glue themselves to the living epidermal tissue of fishes. Flammang et al. (1998) tabulate the total protein present in starfish (A. rubens), limpet pedal mucus (Patella vulgata), barnacle basal cement (Balanus crenatus) and mussel byssal attachment discs (Mytilus edulis). All these adhesives contain a proteinaceous component, but in starfish and limpet it is $21 \%$ and $33 \%$ respectively, whereas in mussel and barnacle the level is much higher (99\% and $84 \%$ respectively). The protein, as a percentage of dry weight, has not been determined for any monogenean adhesive, but histochemistry indicates no lipid or carbohydrate components and solubility via digestion of proteins was complete which suggests that a high protein percentage is present, perhaps differing from starfish and limpet temporary adhesives.

Interestingly, alanine and glycine are often found in large quantities in tightly bound, strong proteins such as those in silk (Alberts et al. 1994). These amino acids are also present at high levels in the SAM of the seven monogenean species studied here. Proteins of similar amino acid content to monogenean SAM are neurophysin and keratin (as identified via an amino acid content search of databases using the ExPASy WWW server). Keratin is a fibrous protein composed of $\alpha$ helices that interlink via disulphide bonds to form coiled-coil dimers (Alberts et al. 1994), being a highly organised, tightly folded structural protein. Long molecules with protein folds compacted by intermediate bonds result in high tensile strength and toughness (Smith et al. 1999b). Such molecules are found in spider dragline silk and nacre from abalone shell and act in such a way that when force rises to a point where it threatens to break a strong bond in the molecular "backbone", a domain unfolds instead, with the process continuing until all the domains are unfolded (Smith et al. 1999b). The strength and impenetrability (as judged by their insolubility) of monogenean anterior adhesives and their proteinaceous nature leads us to speculate that they may also contain long molecules looped into dense modules. Other marine glues appear to be composed of collagen-like proteins made of $\beta$-pleated sheets (Waite et al. 1989, Rzepecki and Waite 1995). Perhaps the regular banding visible using TEM in the rod-like bodies of many monogenean species (see Whittington and Cribb 2001) is indicative of large, tightly packed proteins.

\section{De-adhesion}

In monogenean parasites, there is some evidence that the tegument of the worm may be involved in deadhesion (Kearn and Evans-Gowing 1998). As starfish display a somewhat similar adhesive system, the models for de-adhesion proposed for these echinoderms may be applicable. Thomas and Hermans (1985) suggested that de-adhesion occurs via secreted glycosaminoglycans that competitively displace the glue secreted by the tube-feet of starfish. Heparin, a glycosaminoglycan (highly acidic polysaccharide), prevents adhesion by the starfish Leptasterias hexactis (see Thomas and Hermans 1985). Adhesion was not completely inhibited by heparin in the two monogenean species tested here, but some lessening of adhesion occurred. Perhaps lectin probes may clarify the presence or absence of glycosaminoglycans on newly detached specialised tegument of the anterior adhesive areas of monogeneans. Flammang et al. (1998) present immunocytochemical data that support a second model for deadhesion in starfish (see Flammang 1996) rather than the Thomas and Hermans model. Here, an enzyme releases the fuzzy coat from the surface of the tube foot, 
leaving the fuzzy coat on the glue (Flammang et al. 1998). It is possible that the anterior adhesive area tegument of monogeneans may secrete an enzyme which is responsible for de-adhesion or perhaps the second, granular secretory bodies are involved, although Kearn and Evans-Gowing (1998) found evidence to dismiss the latter possibility, supporting a role for the tegument. Certainly the anterior adhesive area tegument contains different inclusions from the general body tegument (Whittington et al. 2000, Whittington and Cribb 2001). To fully understand the de-adhesion process in monogenean flatworms, it will be necessary to pursue further electron microscopic studies of the anterior adhesive areas before and after release of the glue as has been done for E. soleae by Kearn and
Evans-Gowing (1998). Direct analysis is needed to determine whether changes in the tegument or tegumental secretions occur.

Acknowledgements. The authors thank staff of: the Centre for Drug Design and Development, University of Queensland, for technical assistance, the Centre for Microscopy and Microanalysis, University of Queensland, for access to instrumentation and the Heron Island Research Station of the University of Queensland for hospitality and access to facilities. We also thank Mr. Bob Turner of the Centre for Environmental Fisheries and Aquaculture Science, Lowestoft, UK for supplying soles and Mr. R. Evans-Gowing of the School of Biological Sciences, University of East Anglia, Norwich, UK for technical assistance.

\section{REFERENCES}

ALBERTS B., BRAY D., LEWIS J., RAFF M., ROBERTS K., WATSON J.D. 1994: Molecular Biology of the Cell. Garland Publishing Inc., New York, 1294 pp.

BANCROFT J.D., STEVENS A. 1996: Theory and Practice of Histological Techniques. Churchill Livingstone, New York, $766 \mathrm{pp}$.

BENEDICT C.V., WAITE J.H. 1986: Composition and ultrastructure of the byssus of Mytilus edulis. J. Morphol. 189: 261-270.

COHEN S.A., MICHAUD D.P. 1993: Synthesis of a fluorescent derivatizing reagent, 6-aminoquinolyl-Nhydrosuccinimidyl carbamate, and its application for the analysis of hydrolysate amino acids via high-performance liquid chromatography. Anal. Biochem. 211: 279-287.

CRIBB B.W., WHITTINGTON I.D., CHISHOLM L.A. 1997 Observations on ultrastructure of the anterior adhesive areas and other anterior glands in the monogenean, Monocotyle spiremae (Monocotylidae), from the gills of Himantura fai (Dasyatididae). Int. J. Parasitol. 27: 907917.

CRIBB B.W., WHITTINGTON I.D., CHISHOLM L.A. 1998: Observations on the ultrastructure of the anterior adhesive areas and other anterior gland cells in the monogenean Merizocotyle australensis (Monocotylidae) from the nasal fossae of Himantura fai (Dasyatididae). Microsc. Res. Tech. 42: 200-211.

EL NAGGAR M.M., KEARN G.C. 1983: Glands associated with the anterior adhesive areas and body margins in the skin-parasitic monogenean Entobdella soleae. Int. J. Parasitol. 13: 67-81.

FLAMMANG P. 1996: Adhesion in echinoderms. In: M. Jangoux and J.M. Lawrence (Eds.), Echinoderm Studies. A.A. Balkema, Rotterdam, pp. 1-60.

FLAMMANG P., DEMEULENAERE S., JANGOUX M. 1994: The role of podial secretions in adhesion in two species of sea stars (Echinodermata). Biol. Bull. Mar. Biol. Lab., Woods Hole 187: 35-47.

FLAMMANG P., MICHEL A., VAN CAUWENBERGE A., ALEXANDRES H., JANGOUX M. 1998: A study of the temporary adhesion of the podia in the sea star Asterias rubens (Echinodermata, Asteroidea) through their footprints. J. Exp. Biol. 201: 2383-2385.

HUMASON G.L. 1972: Animal Tissue Techniques. In: R. Emerson, D. Kennedy, R.B. Park, G.W. Beadle and D.M. Whitaker (Eds.), A Series of Books in Biology. W.H. Freeman and Company, San Francisco, 641 pp.

JOHANSSON S.A., SKOOG B. 1987: Rapid silver staining of polyacrylamide gels. J. Biochem. Biophys. Methods 14: 33.

KAMINO K., ODO S., MARUYAMA T. 1996: Cement proteins of the acorn barnacle, Megabalanus rosa. Biol. Bull. Mar. Biol. Lab., Woods Hole 190: 403-409.

KEARN G.C. 1988: Orientation and locomotion in the monogenean parasite Entobdella soleae on the skin of its host (Solea solea). Int. J. Parasitol. 18: 753-759.

KEARN G.C. 1994: Evolutionary expansion of the Monogenea. Int. J. Parasitol. 24: 1227-1271.

KEARN G.C. 1999: The survival of monogenean (platyhelminth) parasites on fish skin. Parasitology 119: S57S88.

KEARN G.C., EVANS-GOWING R. 1998: Attachment and detachment of the anterior adhesive pads of the monogenean (platyhelminth) parasite Entobdella soleae from the skin of the common sole (Solea solea). Int. J. Parasitol. 28: 1583-1593.

LAEMMLI U.K. 1970: Cleavage of structural proteins during the assembly of the head of bacteriophage T4. Nature 227: 680.

MOLLOY M.P., HERBERT B.R., WALSH B.J., TYLER M.I., TRAINI M., SANCHEZ J.-C., HOCHSTRASSER D., WILLIAMS K.L., GOOLEY A.A. 1998: Extraction of membrane proteins by differential solubilization for separations using two-dimensional gel electrophoresis. Electrophoresis 19: 837-844.

NALDRETT M.J. 1993: The importance of sulphur crosslinks and hydrophobic interactions in the polymerisation of barnacle cement. J. Mar. Biol. Assoc. U.K. 73: 689702 .

NALDRETT M.J., KAPLAN D.L. 1997: Characterisation of barnacle (Balanus eburneus and B. cenatus) adhesive proteins. Mar. Biol. 127: 629-635. 
PRESCOTT N. 1997: The production of immunogenic recombinant proteins and protein chimeras from plasmodial merozoite surface proteins. Unpublished $\mathrm{PhD}$ thesis, University of Queensland, Brisbane, $171 \mathrm{pp}$.

RZEPECKI L.M., CHIN S.S., WAITE J.H. 1991: Molecular diversity of marine glues: polyphenolic proteins from five mussel species. Mol. Mar. Biol. Biotechnol. 1: 78-88.

RZEPECKI L.M., WAITE J.H. 1995: Wresting the muscle from mussel beards: research and applications. Mol. Mar. Biol. Biotechnol. 4: 313-322.

SAMBROOK J., FRITSCH E.F., MANIATIS T. 1989: Appendix B: Preparation of reagents and buffers used in molecular cloning. In: Molecular Cloning: a Laboratory Manual. Vol. 3. Cold Spring Harbour Laboratory Press, New York, pp. B.1-B.28.

SMITH A.M., QUICK T.J., PETER R.L.S. 1999a: Differences in the composition of adhesive and non-adhesive mucus from the limpet Lottia limatula. Biol. Bull. Mar. Biol. Lab., Woods Hole 196: 34-44.

SMITH B.L., SCHAFFER T.E., VIANI M., THOMPSON J.B., FREDERICK N.A., KINDT J., BELCHER A., STUCKY G.D., MORSE D.E., HANSMA P.K. 1999b: Molecular mechanistic origin of the toughness of natural adhesives, fibres and composites. Nature 399: 761-763.

THOMAS L., HERMANS C. 1985: Adhesive interactions between the tube feet of a starfish, Leptasterias hexactis, and substrata. Biol. Bull. Mar. Biol. Lab., Woods Hole 169: 675-688.

TYLER S. 1988: The role of function in determination of homology and convergence - examples from invertebrate adhesive organs. Fortschr. Zool. 36: 331-347.

WAITE J.H. 1983: Adhesion in byssally attached bivalves. Biol. Rev. 58: 209-231.

WAITE J.H. 1986: Mussel glue from Mytilus californianus Conrad: a comparative study. J. Comp. Physiol. B 156: 491-496.
WAITE J.H. 1990: The phylogeny and chemical diversity of quinone-tanned glues and varnishes. Comp. Biochem. Physiol. 97B: 19-29.

WAITE J.H., BENEDICT C.V. 1984: Assay of dihydroxyphenylalanine (Dopa) in invertebrate structural proteins. Methods Enzymol. 107B: 397-413.

WAITE J.H., HANSEN D.C., LITTLE K.T. 1989: The glue protein of ribbed mussels (Geukensia demissa): a natural adhesive with some features of collagen. J. Comp. Physiol. B 159: 517-525.

WALKER G. 1987: Marine organisms and their adhesion. In: C.D. Wake (Ed.), Synthetic Adhesives and Sealants. John Wiley \& Sons, Chichester, pp. 112-135.

WHITTINGTON I.D., COMBES C. 1994: Second International Symposium on Monogenea, Montpellier and Sète, France, 5-8 July 1993. Int. J. Parasitol. 24: 445-447.

WHITTINGTON I.D., CRIBB B.W. 1998: Glands associated with the anterior adhesive areas of the monogeneans, Entobdella sp. and Entobdella australis (Capsalidae) from the skin of Himantura fai and Taeniura lymma (Dasyatididae). Int. J. Parasitol. 28: 653-665.

WHITTINGTON I.D., CRIBB B.W. 1999: Morphology and ultrastructure of the anterior adhesive areas of the capsalid monogenean parasites Benedenia rohdei from the gills and B. lutjani from the pelvic fins of Lutjanus carponotatus (Pisces: Lutjanidae). Parasitol. Res. 85: 399-408.

WHITTINGTON I.D., CRIBB B.W., HAMWOOD T.E., HALLIDAY J.A. 2000: Host-specificity of monogenean (platyhelminth) parasites: a role for anterior adhesive areas. Int. J. Parasitol. 30: 305-320.

WHITTINGTON I.D., CRIBB B.W. 2001: Adhesive secretions in the Platyhelminthes. Adv. Parasitol. 48: 101224.

Accepted 31 May 2001 